\title{
The role of self-motion in acrophobia treatment
}

Carlos M. Coelho, Jorge A. Santos, Carlos Silva, Guy Wallis, Jennifer Tichon, Trevor J. Hine

CM Coelho, $\mathrm{PhD}$ is a Senior Research Fellow in the School of Human Movement Studies, University of Queensland, Level 5, Building 26, St Lucia Queensland Australia 4072.

Address all correspondence to Dr Coelho at: ccoelho@hms.uq.edu.au

JA Santos, $\mathrm{PhD}$, is an Associate Professor in the Department of Psychology, University of Minho, Campus de Gualtar 4710 - 057 Braga Portugal

CF da Silva, PhD, is a Professor in the Department of Education Sciences, University of Aveiro, 3810-193 Aveiro Portugal

$\mathrm{G}$ Wallis, PhD is an ARC Senior Research Fellow in the School of Human Movement Studies, University of Queensland

J Tichon, PhD, is a Senior Research Fellow in the School of Human Movement Studies, University of Queensland

TJ Hine, PhD, is a Senior Lecturer in the School of Psychology and a member of the Applied Cognitive Neuroscience Research Centre, Griffith University, Mt Gravatt Queensland Australia 
Abstract: Acrophobia is a chronic highly debilitating disorder preventing sufferers from engaging with high places. Its aetiology is linked to the development of mobility during infancy. We evaluated the efficacy of various type of movement in the treatment of this disorder within a virtual reality environment. Four men and four women who were diagnosed with acrophobia were tested in a virtual environment reproducing the balcony of a hotel. Anxiety and behavioral avoidance measures were taken as participants climbed outdoor stairs, moved sideways on balconies or stood still. This took place in both a real and virtual environment as part of a treatment evaluation study. Participants experienced an elevated level of anxiety not only to increases in height, but also when required to move laterally at a fixed height. These anxiety levels were significantly higher than those elicited by viewing the fear-invoking scene without movement. We have demonstrated a direct link between any type of movement at a height and the triggering of acrophobia in line with earlier developmental studies. Given this is suggested that recalibration of the action-perception system, aided by virtual reality, can be an important adjunct to standard psychotherapy. 


\section{Introduction}

Studies of the treatment of acrophobia have traditionally focussed on the effects of different therapeutic approaches ${ }^{1}$, cognitive processes ${ }^{2}$ and the time and frequency of the exposure treatments ${ }^{3}$. Despite its apparent etiological importance ${ }^{4}$, motion is almost never mentioned as an important factor in exposure therapy. Nevertheless, the close relationship between perception and motor activity is well established. We know, for example, that postural control is dependent upon the integration of information from the proprioceptive, vestibular and visual sensory systems $^{5}$. If preference is given to visual inputs, the individuals become sensitive to perturbations in their visual surroundings ${ }^{6}$. This increased reliance on vision may also be reflected in complaints of disequilibrium during certain situations of daily living that include complex, moving visual environments or heights ${ }^{7}$. In the present study, we hypothesize that the level of fear experienced by participants is related to the level of self motion, as opposed to being determined by an exposure to a particular height. In line with this hypothesis, this study focuses on the subjective evaluation of discomfort during three levels of movement: 1) the participant is static, with no noticeable head or body movements; 2) he or she is walking along at a particular level and turning his or her head to explore the environment; or 3) he or she is going up to a higher level in the virtual world.

\section{Method}

Data from 8 participants, 4 men and 4 women with ages ranging between 23 and 58 (mean 32.7) who took part in a broader study of acrophobia treatment ${ }^{8}$ were collected. The participants were first informed about the exposure procedures, and their informed written consent was obtained. Subjects received a pre-test followed by 
three sessions of VR exposure. Sessions lasted about 35 minutes and were held weekly. As measures of acrophobia we used the Subjective Units of Discomfort (SUD) and a Behavioral Avoidance Test (BAT). The software rendered a scene visualized with a helmet mounted display and a tracker which recreated the view from a balcony. The participants' level of anxiety was assessed with a SUD score every three minutes. Also, all significant corrective steps and body movements were recorded on a scale from 1 to 10 , providing a record of subjective discomfort.

\section{Data Analysis}

SUD scores were measured as the participants experienced one of three categories of motion. These categories involved the participant: 0 ) being static, with no noticeable head or body movements; 1) walking along the balcony and moving the head to explore the environment; 2) climbing to a higher balcony in the virtual world.

\section{Results}

A non-parametric Kruskal-Wallis analysis compared SUD values by the three types of movement across all participants and revealed statistically significant differences among the three $\left(\chi^{2}=71.255 ; \mathrm{p}<.001\right)$. Comparing horizontal (type 1) movements SUD with the static type 0 (number of observations $=25$ ), the non parametric Wilcoxon test revealed a $\mathrm{Z}=-3,401$ and $\mathrm{p}=.001$. The SUD in Type 2 movements, compared with type 0 (number of observations $=21$ ), Wilcoxon revealed a $\mathrm{Z}=-3,394 ; \mathrm{p}=.001$. An example of the relation between types of movement and anxiety can be seen in Figure 1.

Figure 1 -Participant 4 movement type during treatment and SUDS scores during three minute epochs 


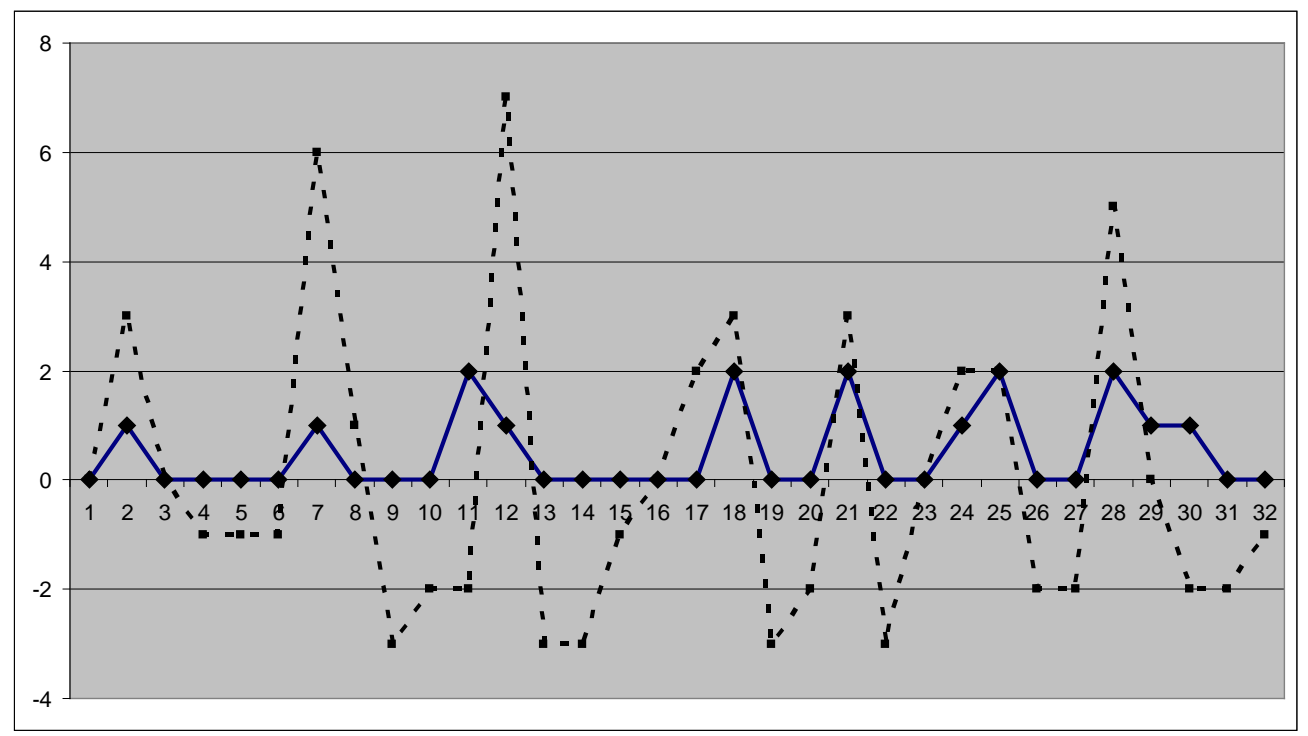

Legend: $y$ axe dotted line $=$ SUDS; $y$ axe continuous line $=$ kind of movement $(0=$ no movement $)$; (1=horizontal displacements); ( 2 = climbing up the virtual building stairs

Data were plotted for each participant and the overall mean for each movement type and compared with variations in the SUD score. Plots of the data suggest a strong relationship among the three kinds of movement (types 0,1 and 2) and the anxiety of the participants in the virtual world measured by SUD. It is clear that the median SUD ratios are similar for both movement types - 1 (horizontal displacement) and 2 (climbing up).

\section{Discussion}

We found that average SUD scores are similar for both horizontal displacement (type 1) and climbing up (type 2) movements, but both are greater than just static exposure (type 0 ). Moreover, the variance in these scores for the type 1 movement was smaller than for type 2. Therefore, it seems that exploratory patterns of the environment, such as walking around, postural adjustments and head movements, do promote more consistent and higher rates of fear than vertical climbing 
movements. We also found that the magnitude of variation in the SUD scores correlate with the type of movement performed by participants. This effect was noticed mainly in the first session, after which habituation occurred. Responses during treatment are not able to identify the etiological factors affecting acrophobia, but they do support the idea that movement is an important factor in creating fear responses, which appeared to relate to perceived violations of the expected correlation between visual, vestibular and somatosensory inputs. This is consistent with research regarding fear reactions in children tested on the visual cliff $^{9}$ as well with findings showing that exercises to promote awareness of somatosensory inputs enhances the participant's confidence in height situations $^{6}$. Further studies are needed to corroborate and extend these findings; namely, to highlight what kind of head and body movements and which linear or angular amplitudes are crucial as phobia triggers.

\section{Conclusion}

We consider our framework relevant not only from fundamental and psychopathologic perspectives but also for clinical applications. Clinicians usually consider the variable "height" as the main, if not the only, feature of the exposure therapy for acrophobia. Our study highlights the importance of self-motion in acrophobia. We would suggest that it is important for the clinician to train his/her clients to perform several movements in balconies or other elevated environments, which will not only elicit the levels of fear required to accomplish habituation, but also provide improved sensory training to ameliorate the root causes of the disorder.

\section{ACKNOWLEDGEMENTS}

This research was funded by a Science and Technology Foundation Grant (ref. SFRH/BPD/21977/2005) 


\section{Reprint requests should be directed to}

Carlos M. Coelho

School of Human Movement Studies

University of Queensland, Level 5, Building 26, St Lucia

Queensland Australia 4072.

ccoelho@hms.uq.edu.au

\section{References}

1 Williams SL, Turner SM, Peer DF. Guided mastery and performance desensitization for severe acrophobia. J Consult Clin Psychol. 1985; 53: 237-247.

2 Marshall WL, Bristol D, Barbaree HE. Cognitions and courage in the avoidance behavior of acrophobics. Behav Res Ther. 1992; 30: 463-470.

3 Marshall WL. The effects of variable exposure to flooding therapy. Behav Res Ther. 1985; 16: 117-135.

4 Campos JJ, Hiatt S, Ramsay D, Henderson C, Svejda M: The emergence of fear on the visual cliff. In: Lewis M, L.A. R, eds. The development of affect. New York: Plenum Press, 1978; 149-182.

5 Redfern MS, Yardley L, Bronstein AM. Visual Influences on Balance. J Anxiety Disord. 2001; 15: 81-94.

6 Whitney SL, Jacob RG, Sparto PJ, et al. Acrophobia and pathological height vertigo: Indications for vestibular physical therapy? Phys Ther. 2005; 85: 443-458.

7 Adkin A, L., Frank JS, Carpenter MG, Peysar GW. Postural control is scaled of postural threat. Gait and Posture. 2000; 12: 87-93.

8 Coelho, C.M., Santos, J.A. Silvério, J. \& Silva, C.F. (2006) Virtual Reality and Acrophobia: One-Year Follow-Up and Case Study. CyberPsychology \& Behavior, 9, 336-341.

9 Campos JJ, Anderson DI, Barbu-Roth MA, Hubbard EM, Hernstein MJ, Witherington D. Travel broadens the mind. Infancy. 2000; 1: 149-219. 